\title{
Common zeroes of families of smooth vector fields on surfaces
}

\author{
Morris W. Hirsch Mathematics Department \\ University of Wisconsin at Madison \\ University of California at Berkeley
}

September 6, 2018

\begin{abstract}
Let $Y$ and $X$ denote $C^{k}$ vector fields on a possibly noncompact surface with empty boundary, $1 \leq k<\infty$. Say that $Y$ tracks $X$ if the dynamical system it generates locally permutes integral curves of $X$. Let $K$ be a locally maximal compact set of zeroes of $X$.

Theorem. Assume the Poincare-Hopf index of $X$ at $K$ is nonzero, and the $k$-jet of $X$ at each point of $K$ is nontrivial. If $\mathfrak{g}$ is a supersolvable Lie algebra of $C^{k}$ vector fields that track $X$, then the elements of $\mathfrak{g}$ have a common zero in $K$.

Applications are made to attractors and transformation groups.
\end{abstract}

\section{Contents}

1 Introduction 1

Statement of the main results . . . . . . . . . . . . 2

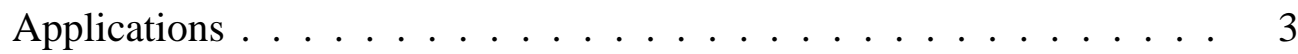

2 Index calculations 5

3 Proof of Theorem $1.2 \quad 6$

\section{Introduction}

$M$ denotes a metrizable real analytic surface with empty boundary. The vector space of vector fields on $M$ is $\mathcal{V}(M)$, topologized by uniform convergence on compact sets. The subspace of $C^{r}$ vector fields is $\mathcal{V}^{r}(M)$. Here $r \in \mathbb{N}_{+}$(the set of positive integers), or $r=\infty$ (infinitely differentiable), or $r=\omega$ (analytic). 
$X$ denotes a vector field on $M$, with zero set $\mathrm{Z}(X)$. A compact set $K \subset \mathrm{Z}(X)$ is a block of zeroes for $X$, or an $X$-block, if it has a precompact open neighborhood $U \subset M$ such that $\mathrm{Z}(X) \cap \bar{U}=K$. We say $U$ is isolating for $X$ and for $(X, K)$.

The index of the $X$-block $K$ is the integer $\mathrm{i}_{K}(X):=\mathrm{i}(X, U)$ defined as the Poincaré-Hopf index $[20,13]$ of any sufficiently close approximation to $X$ having only finitely many zeroes in $U$. This number is independent of $U$, and is stable under perturbations of $X$ : If $Y \in \mathcal{V} M$ is sufficiently close to $X$ then $U$ is isolating for $Y$, and $\mathrm{i}(Y, U)=\mathrm{i}(X, U)\left(\right.$ Proposition 2.2). ${ }^{1}$

$K$ is essential if $\mathrm{i}_{K}(X) \neq 0$, which every isolating neighborhood of $K$ meets $\mathrm{Z}(X)$. This powerful condition implies that if $Y$ is sufficiently close to $X$ then $\mathrm{Z}(Y) \cap U \neq \varnothing$.

We say $Y$ tracks $X$ provided $X, Y \in \mathcal{V}^{1}(M)$, and the corresponding local flows $\Phi^{Y}=\left\{\Phi_{t}^{Y}\right\}_{t \in \mathbb{R}}$ and $\Phi^{X}=\left\{\Phi_{t}^{X}\right\}_{t \in \mathbb{R}}$ have the following property: For each $t \in \mathbb{R}$ the $C^{1}$ diffeomorphism $\Phi_{t}^{Y}: \mathcal{D}_{t} \approx \mathcal{R}_{t}$ maps orbits of $X \mid \mathcal{D}_{t}$ to orbits of $X \mid \mathcal{R}_{t}$. Equivalently: There exists $f: M \backslash Z(X) \rightarrow \mathbb{R}$ such that $X_{p} \neq 0 \Longrightarrow[Y, X]=f(p) X_{p}$ (see Hirsch, [12, Prop. 2.4] or [10, Prop. 2.3]). ${ }^{2}$

If $X$ spans an ideal in a Lie algebra $\mathfrak{g} \subset \mathcal{V}^{k}(M)$, every element of $\mathfrak{g}$ tracks $X$. When $X$ is $C^{\infty}$, the set of $Y \in \mathcal{V}^{\infty}(M)$ that track $X$ is an infinite dimensional Lie algebra.

\section{Statement of the main results}

Throughout the rest of this article we assume:

- $X \in \mathcal{V}^{k}(M), k \in \mathbb{N}_{+}$.

$X$ has $\operatorname{order} \operatorname{ord}_{p}(X):=j \in\{1, \ldots, k\}$ at $p \in Z(X)$ if $j$ is the smallest number in $\{1, \ldots, k\}$ such that the $j$-jet of $X$ at $p$ is nontrivial. In other words: Some (and hence every) $C^{k+1}$ chart $M \supset W^{\prime} \approx W \subset \mathbb{R}^{2}$ on $M$, centered at $p$, represents $X \mid W$ by a $C^{k}$ map $F: W \rightarrow \mathbb{R}^{2}$ whose partial derivatives at the orgin satisfy

$$
F^{(i)}(0)=0 \text { for } i=0, \ldots, j-1, \quad F^{(j)}(0) \neq 0 .
$$

If no integer $j$ has this property, $X$ is $k$-flat at $p$.

When $X$ is analytic and nontrivial on a neighborhood of a continuum $L \subset Z(X)$, the order of $X$ is constant on $L$.

Theorem 1.1. Assume $X, Y \in \mathcal{V}^{k}(M)$ have the following properties:

(a) $K \subset Z(X)$ is an essential $X$-block,

(b) $X$ is not $k$-flat at any point of $K$,

(c) $Y$ tracks $X$.

\footnotetext{
${ }^{1}$ Equivalently: $\mathrm{i}(X, U)$ is the intersection number of $X \mid U$ with the zero section of the tangent bundle (C. BonatTi [3]). If $X$ is generated by a smooth local flow $\phi$ then $\mathrm{i}(X, U)$ equals the fixedpoint index $I\left(\phi_{t} \mid U\right)$ of A. Dold [5] for sufficiently small $t>0$.

${ }^{2}$ Article [10] is a preliminary version of [12].
} 
Then $\mathrm{Z}(Y) \cap K \neq \varnothing$.

When $X$ and $Y$ are commuting analytic vector fields, this is a special case of a remarkable theorem of C. BonATTI [3] — the inspiration for the present paper.

Theorem 1.1 is proved by demonstrating the strong form of the contrapositive stated below.

A line field $\Lambda$ on a set $N \subset M$ is a (continuous) section $p \mapsto \Lambda_{p}$ of the fibre bundle over $N$ whose fibre over $p \in N$ is the circle of unoriented lines through the origin in the tangent space $T_{p}(M)$. If $Y_{p} \in \Lambda_{p}$ for all $p \in N$ then $\Lambda$ controls $Y$ in $N$.

Theorem 1.2. Assume:

(a) $X \in \mathcal{V}^{k}(M)$ is not $k$-flat at any point of the $X$-block $K$,

(b) $Y \in \mathcal{V}^{k}(M)$ tracks $X$,

(c) $\mathrm{Z}(Y) \cap K=\varnothing$,

(d) $U \subset M$ is an isolating neighborhood for $(X, K)$.

Then:

(i) $\mathrm{i}_{K}(X)=0$.

(ii) K has only finitely many components, and each component is a $C^{k}$-embedded circle.

(iii) $X$ is controlled by a unique line field in $U$.

(iv) $X$ has index zero at each component of $K$.

(v) $X$ can be $C^{k}$-approximated by vector fields that have no zeroes in $U$ and agree with $X$ outside $U$.

The is in Section 3.

\section{Applications}

Let $\mathfrak{g} \subset \mathcal{V}^{k}(M)$ denote a Lie algebra- a linear subspace closed under Lie brackets. The zero set of $\mathfrak{g}$ is defined as $\mathrm{Z}(\mathfrak{g}):=\bigcap_{Y \in \mathfrak{g}} \mathrm{Z}(Y)$. If every $Y \in \mathfrak{g}$ tracks $X$, then $\mathfrak{g}$ tracks $X$. We call $\mathfrak{g}$ supersolvable if it is faithfully represented by upper triangular real matrices.

Theorem 1.3. Assume:

(a) $K$ is an essential $X$-block,

(b) $X$ is not $k$-flat at any point of $K$,

(c) $\mathfrak{g} \subset \mathcal{V}^{k}(M)$ is a supersolvable Lie algebra tracking $X$. 
Then $\mathrm{Z}(\mathfrak{g}) \cap K \neq \varnothing$.

Related theorems and counterexamples are discussed in HIRSCH [12].

Theorem 1.4. Suppose the local flow of $X \in \mathcal{V}^{\omega}(M)$ has a compact attractor $P \subset M$ with Euler characteristic $\chi(P) \neq 0$. Then there exists $k \in \mathbb{N}_{+}$with the following property: If $\mathfrak{h} \subset \mathcal{V}^{k}(M)$ is a supersolvable Lie algebra that tracks $X$, then $\mathrm{Z}(\mathfrak{h}) \cap \mathrm{Z}(X) \cap P \neq \varnothing$.

Proof. If $P=M$ then $M$ is a closed surface and $\chi(M) \neq 0$. Therefore $\mathrm{Z}(X)$ is an essential $X$-block by Poincaré's Theorem [20], and the conclusion follows from Theorem 1.2.

Suppose $P \neq M$. The basin of attraction of $P$ contains a smooth compact surface $N$ with boundary such that $Y$ is inwardly transverse to $\partial N$. The interior $N_{0}:=N \backslash \partial N$ is a precompact open set which is positively invariant under $\Phi^{Y}(\mathrm{~F}$. WILson [25, Th. 2.2]). Therefore

$$
t>s \geq 0 \Longrightarrow \Phi_{t}^{Y}\left(N_{0}\right) \subset \Phi_{s}^{Y}\left(N_{0}\right), \quad \bigcap_{t \geq 0} \Phi_{t}^{Y}\left(N_{0}\right)=P .
$$

The inclusion maps $P \hookrightarrow N_{0} \hookrightarrow N$ induce isomorphisms of Čech cohomology groups, hence $\chi\left(N_{0}\right) \neq 0$. The conclusion follows from Theorem 1.3 appplied to $X:=Y \mid N_{0}$ and the Lie algebra $\mathfrak{g} \subset \mathcal{V}^{k}\left(N_{0}\right)$ comprising the restrictions of the vector fields in $\mathfrak{h}$ to $N_{0}$.

Example 1.5. Assume $P \subset \mathbb{R}^{2}$ is a compact global attractor for $X \in \mathcal{V}^{\omega}\left(\mathbb{R}^{2}\right)$ and $\mathfrak{h} \subset \mathcal{V}^{k}\left(\mathbb{R}^{2}\right)$ is a supersolvable Lie algebra tracking $Y$. Then:

- $\mathrm{Z}(\mathfrak{h}) \cap \mathrm{Z}(X) \cap P \neq \varnothing$.

Proof. $\chi(P) \neq 0$ because $\mathbb{R}^{2}$ is contractible and $P$ is a global attractor, so Theorem 1.4 yields the conclusion.

Corollary 1.6. Let $G$ be a connected Lie group whose Lie algebra is supersolvable, with an effective $C^{\infty}$ action on a compact surface $M$. If $\chi(M) \neq 0$ and the action is analytic on a normal 1-dimensional Lie subgroup, then G fixes a point of $M$.

The special case in which $M$ is compact and $G$ acts analytically is due to HIRSCH \& WeINSTEIN [7].

Proof. The action of $G$ on $M$ induces an isomorphism $\theta$ from the Lie algebra $\mathfrak{a}$ of $G$ onto a subalgebra $\mathfrak{g} \subset \mathcal{V}^{\infty}(M)$. Let $Y \in \mathfrak{a}$ span the Lie algebra of $H$ and set $\theta(Y)=X \in \mathfrak{g}$. Then $X \in \mathcal{V}^{\omega}(M)$ and $\mathfrak{g}$ tracks $X$, whence the conclusion from Theorem 1.4.

Related results on Lie group actions and Lie algebras of vector fields can be found in the articles $[1,2,4,8,9,11,15,17,18,19,22,23,24]$. 


\section{Index calculations}

Proposition 2.1. Assume $Y, X \in \mathcal{V}^{k}(M)$ and $Y$ tracks $X$.

(i) $\mathrm{Z}(X)$ is invariant under $\Phi^{Y}$.

(ii) If $p, q \in \mathrm{Z}(X)$ are in the same orbit of $\Phi^{Y}$, then $\operatorname{ord}_{p}(X)=\operatorname{ord}_{q}(X)$.

Proof. Follows from the definition of tracking.

These properties of the index function are crucial:

Proposition 2.2 (Stability). Let $U \subset M$ be isolating for $X$.

(a) If $\mathrm{i}(X, U) \neq 0$ then $\mathrm{Z}(X) \cap U \neq \varnothing$.

(b) If $Y$ is sufficiently close to $X$ then $\mathrm{i}(Y, U)=\mathrm{i}(X, U)$.

(c) Let $\left\{X^{t}\right\}_{t \in[0,1]}$ be a deformation of $X$. If each $X^{t}$ is nonsingular on the frontier of $U$, then $\mathrm{i}\left(X^{t}, U\right)=\mathrm{i}(X, U)$.

Proof. This is Theorem 3.9 of [12].

Proposition 2.3. Assume $Y, Y^{\prime} \in \mathcal{V}(M)$ and $U \subset M$ is isolating for both $Y$ and $Y^{\prime}$. Assume $N:=\bar{U}$ is a compact $C^{1}$ surface such that $Y_{p}$ and $Y_{p}^{\prime}$ are linearly dependent at all $p \in \partial N$. Then $\mathrm{i}(Y, U)=\mathrm{i}\left(Y^{\prime}, U\right)$.

Proof. This consequence of Proposition 2.2 is a special case of [10, Prop. 3.12] or [12, Prop. 3.11].

Proposition 2.4. Let $K \subset M$ be a block of zeroes for $X \in \mathcal{V}^{k}(M)$, and $U \subset M$ an isolating neighborhood for $(X, K)$. If $X \mid U$ is controlled by a line field $\Lambda$ on $U$, then $\mathrm{i}_{K}(X)=0$.

Proof. By shrinking $U$ slightly, we assume $N:=\bar{U}$ is a compact $C^{1}$ surface.

Suppose $\Lambda$ is an orientable line field. Then $\Lambda$ controls a nonsingular vector field $Y$ on $N$, and $\mathrm{i}(Y, U)=0$ because $\mathrm{Z}(Y)=\varnothing$. Evidently $U$ is isolating for both $X$ and $Y$, and $X_{p}, Y_{p}$ are linearly dependent at all $p \in N$. Proposition 2.3 implies $\mathrm{i}(X, U)=\mathrm{i}(Y, U)=0$, hence $\mathrm{i}_{K}(X)=0$.

Now suppose $\Lambda$ is nonorientable. There is a double covering $\pi: \tilde{V} \rightarrow V$ of an open neighborhood $V \subset M$ of $N$, isolating for $(X, K)$, such that $\Lambda$ lifts to an orientable line field on $\tilde{V}$. The orientable case shows that the vector field $\tilde{X}$ on $\tilde{V}$ that projects to $X \mid V$ under $\pi$ has index zero in $\tilde{V}$.

Fix $X_{1} \in \mathcal{V}(V)$ with $\mathrm{Z}\left(X_{1}\right)$ finite, and such that the sum of the Poincaré-Hopf indices of the zeroes of $X_{1}$ equals $\mathrm{i}(X, V)$. Define $\tilde{X}_{1} \in \mathcal{V}(\tilde{V})$ to be the vector field projecting to $X_{1}$ under $\pi$. Each zero $p$ of $X_{1}$ is the image under $\pi$ of exactly two zeroes $q_{1}, q_{2}$ of $\tilde{X}_{1}$, and the Poincaré-Hopf indices of $\tilde{X}$ at $q_{1}$ and $q_{2}$ both equal the Poincaré-Hopf index of $X$ at $p$. Therefore

$$
0=\mathrm{i}\left(\tilde{X}_{1}, \tilde{V}\right)=2 \mathrm{i}\left(X_{1}, V\right)=2 \mathrm{i}(X, V)=2 \mathrm{i}_{K}(X),
$$

completing the proof.

Other calculations of indices can be found in $[5,6,14,16,21]$. 


\section{Proof of Theorem 1.2}

Lemma 3.1. Let $L \subset K$ be a component. Then $X$ has the same order at each point of $L$.

Proof. The sets $L_{j}=\left\{p \in L: \operatorname{ord}_{p}(X)=j\right\}, j \in\{1, \ldots, k\}$ are relatively open in $L$ and mutually disjoint. As $L$ is connected and covered by the $L_{j}$, it coincides with one of them.

Let $p \in K$ be arbitrary. Choose a $C^{k+1}$ flowbox $h_{p}$ for $Y$ centered at $p$ :

$$
h_{p}: W_{p}^{\prime} \approx W_{p}=J_{p} \times J_{p}^{\prime} \subset \mathbb{R}^{2}, \quad h(p)=(0,0) .
$$

This means $W_{p}^{\prime}$ is open in $M, J_{p}, J_{p}^{\prime} \subset \mathbb{R}$ are open intervals around 0 , and the $C^{k+1}$ diffeomorphism $h_{p}$ transforms $Y \mid W_{p}^{\prime}$ to the constant vector field $\frac{\partial}{\partial x} \mid W_{p}$, where $x, y$ are the usual planar coordinates. Notice that $h_{p}\left(K \cap W_{p}^{\prime}\right)=J_{p} \times\{0\}$ because $K$ is $Y$-invariant.

The transform of $X \mid W_{p}^{\prime}$ by $h_{p}$ is a $C^{k}$ vector field

$$
\hat{X}(p) \in \mathcal{V}^{k}\left(W_{p}\right), \quad(x, y) \mapsto F_{p}(x, y),
$$

where $F_{p}: W_{p} \rightarrow \mathbb{R}^{2}$ is $C^{k}$.

Set $\operatorname{ord}_{p}(X)=l \in\{1, \ldots, k\}$. The partials of $F_{p}$ satisfy

$$
F_{p}^{(i)}(0)=0 \text { for } i=0, \ldots, l-1, \quad F_{p}^{(l)}(0) \neq 0 .
$$

In a sufficiently small open disk

$$
D:=D_{p} \subset \mathbb{R}^{2}, \quad(0,0) \in D_{p},
$$

the $l^{\prime}$ th order Taylor expansion of $F_{p}$ about $(0,0)$ takes the form

$$
F_{p}(x, y)=y^{l} g(x, y), \quad g(x, y) \neq(0,0)
$$

with $g: D_{p} \rightarrow \mathbb{R}^{2}$ continuous. Therefore

$$
\mathrm{Z}(\hat{X}(p))=F_{p}^{-1}(0,0)=J_{p} \times\{0\},
$$

whence $K \cap W_{p}^{\prime}$ is an open arc, relatively closed in $W_{p}^{\prime}$.

It follows that $K$ has an open cover by open arcs. Thus $K$ is a compact 1manifold having only finitely many components, each of which is a topological circle. The restriction of $\Phi^{Y}$ to any component $L \subset K$ is a smooth flow with no fixed points. Therefore $L$ is a periodic orbit of $\Phi^{Y}$, and is thus a smooth submanifold. This proves Theorem 1.2(ii).

Lemma 3.2. $\hat{X}$ is controlled by a unique line field $\Lambda(p)$ on $D_{p}$. 
Proof. Consider the unit vector field $\hat{F}$ on $D^{\prime}:=D \backslash J \times\{0\}$, as

$$
\hat{F}(x, y):=\operatorname{sign}(y) \frac{F(x, y)}{\|F(x, y)\|}
$$

where $\|\cdot\|$ denotes the Euclidean norm. Equation (4) implies

$$
\lim _{y \rightarrow 0} \hat{F}(x, y)=\frac{g(x, 0)}{\|g(x, 0)\|} \text { uniformly in } D^{\prime} .
$$

Therefore $\hat{F}$ extends to a unique continuous map $\tilde{F}: D \rightarrow \mathbf{S}^{1}$ (the unit circle). The desired line field sends $(x, y) \in D$ to the line through $(0,0)$ spanned by $\tilde{F}_{(x, y)}$.

Next we prove 1.2(iii). For each $p \in K$ let $V_{p}:=h_{p}^{-1}\left(D_{p}\right)$, with notation as in Equations (2), (3). Define $V:=\bigcap_{p \in K} V_{p}$. Let $\Lambda(p)$ be the line field defined in Lemma 3.2. The pullback of $\Lambda(p)$ by $h_{p}$ is the unique line field $\Gamma(p)$ on $V_{p}$ controlling $X \mid V_{p}$. The unique line field $\Gamma$ on $V$ that restricts to $\Gamma(p)$ for each $p \in K$ has the required properties.

Parts (i) and (iv) of 1.2 are consequences of (iii) and Proposition 2.4.

Part (v) follows from Propositions 3.13 and 3.14 of [12] when $U$ is connected, and this implies the general case because the compact set $K$ is covered by finitely many components of $U$.

\section{References}

[1] M. Belliart, Actions sans points fixes sur les surfaces compactes, Math. Z. 225 (1997), 453-465

[2] M. Belliart \& I. Liousse, Actions affines sur les surfaces, Publications IRMA, Universite de Lille, 38 (1996) exposé X

[3] C. Bonatti, Champs de vecteurs analytiques commutants, en dimension 3 ou 4: existence de zéros communs, Bol. Soc. Brasil. Mat. (N. S.) 22 (1992), 215-247

[4] A. Borel, Groupes linéaires algebriques, Ann. Math. 64 (1956), 20-80

[5] A. Dold, "Lectures on Algebraic Topology," Die Grundlehren der matematischen Wissenschaften Bd. 52, second edition. Springer, New York 1972

[6] D. Gottlieb, A de Moivre like formula for fixed point theory, in: "Fixed Point Theory and its Applications (Berkeley, CA, 1986)." Contemporary Mathematics 72 Amer. Math. Soc., Providence, RI 1988

[7] M. Hirsch \& A. Weinstein, Fixed points of analytic actions of supersoluble Lie groups on compact surfaces, Ergod. Th. Dyn. Sys. 21 (2001), 1783-1787

[8] M. Hirsch, Actions of Lie groups and Lie algebras on manifolds, in "A Celebration of the Mathematical Legacy of Raoul Bott." Centre de Recherches Mathématiques, U. de Montréal. Proceedings \& Lecture Notes 50, (P. R. Kotiuga, ed.), Amer. Math. Soc. Providence RI 2010 
[9] M. Hirsch, Smooth actions of Lie groups and Lie algebras on manifolds, J. Fixed Point Th. App. 10 (2011), 219-232

[10] M. Hirsch, Zero sets of Lie algebras of analytic vector fields on real and complex 2-manifolds, http://arxiv.org/abs/1310.0081 (2013)

[11] M. Hirsch, Fixed points of local actions of nilpotent Lie groups on surfaces, http://arxiv.org/abs/1405.2331 (2014)

[12] M. Hirsch, Zero sets of Lie algebras of analytic vector fields on real and complex 2-manifolds, submitted (2015)

[13] H. Hopf, Vektorfelder in Mannifgfaltigkeiten, Math. Annalen 95 (1925), 340-367

[14] B. Jubin, A generalized Poincaré-Hopf index theorem,(2009) http://arxiv.org/abs/0903.0697

[15] E. Lima, Common singularities of commuting vector fields on 2-manifolds, Comment. Math. Helv. 39 (1964), 97-110

[16] M. Morse, Singular Points of Vector Fields Under General Boundary Conditions, Amer. J. Math. 52 (1929), 165-178

[17] J. Plante, Fixed points of Lie group actions on surfaces, Erg. Th. Dyn. Sys. 6 (1986), 149-161

[18] J. Plante, Lie algebras of vector fields which vanish at a point, J. London Math. Soc (2) 38 (1988) 379-384

[19] J. Plante, Elementary zeros of Lie algebras of vector fields, Topology 30 (1991) 215-222

[20] H. Poincaré, Sur les courbes définies par une équation différentielle, J. Math. Pures Appl. 1 (1885), 167-244

[21] C. Pugh, A generalized Poincaré index formula, Topology 7 (1968), 217-226

[22] A. Sommese, Borel's fixed point theorem for Kaehler manifolds and an application, Proc. Amer. Math. Soc. 41 (1973), 51-54.

[23] F.-J. Turiel, An elementary proof of a Lima's theorem for surfaces, Publ. Mat. 3 (1989) 555-557

[24] F.-J. Turiel, Analytic actions on compact surfaces and fixed points, Manuscripta Mathematica 110 (2003), 195-201

[25] F. W. Wilson, Smoothing derivatives of functions and applications, Trans. Amer. Math. Soc. 139 (1969), 413-428 